\title{
CONSTRUCTION AND ANALYSIS OF AN AUGMENTED LATTICE SQUARE \\ DESIGN
}

\section{Walter T. Federer \\ Dept. of Biometrics Cornell University}

Keywords: none provided.

\begin{abstract}
Augmented designs are useful for screening experiments involving large numbers of new and untried treatments. Since resolvable row-column designs are useful for controlling extraneous variation, it is desirable to use such designs for the check or standard treatments. A simple procedure is described for constructing augmented lattice square designs using $\mathrm{c}=2 \mathrm{k}$ and $\mathrm{c}=3 \mathrm{k}$ check treatments and $\mathrm{n}$ $=\mathrm{rk}(\mathrm{k}-2)$ and $\mathrm{n}=\mathrm{rk}(\mathrm{k}-3)$ new treatments respectively, $\mathrm{r}$ being the number of complete blocks. A trend analysis of these designs is presented. A numerical example is used to describe the analysis. A SAS computer code and the output from this code are included.
\end{abstract}




\title{
CONSTRUCTION AND ANALYSIS OF AN AUGMENTED LATTICE SQUARE DESIGN
}

by

\author{
Walter T. Federer
}

\begin{abstract}
Augmented designs are useful for screening experiments involving large numbers of new and untried treatments. Since resolvable row-column designs are useful for controlling extraneous variation, it is desirable to use such designs for the check or standard treatments. A simple procedure is described for constructing augmented lattice square designs using $c=2 k$ and $c=3 k$ check treatments and $n=r k(k-2)$ and $n=r k(k-3)$ new treatments respectively, $\mathrm{r}$ being the number of complete blocks. A trend analysis of these designs is presented. A numerical example is used to describe the analysis. A SAS computer code and the output from this code are included.
\end{abstract}

BU-1485-M

June 2000

\section{INTRODUCTION}

Augmented experiments designs (AEDs) for screening large numbers of new and untried treatments are in use around the world, especially in plant breeding investigations. AEDs have several advantages over a frequently used plant breeding arrangement involving a single check variety systematically spaced every $s^{\text {th }}$ plot. .Advantages are:

(i) More than one check treatment may be included.

(ii) Standard errors of a difference between two new or augmented treatments are available.

(iii) Standard errors of difference between new and check treatments are available.

(iv) Using the survivors of previous screening as part or all of the checks is a device for testing these survivors at the same time a new set of treatments is being screened.

(v) Fewer cycles of screening and selections are needed than for the systematically spaced single check treatment.

An augmented lattice square design (ALSD) is an experiment design for c checks or standards replicated $r$ times in a $k$ by $k$ lattice in each of the $r$ replicates (complete 
blocks). When $\mathrm{c}=2 \mathrm{k}$, the number of new treatments that may be included can be $\mathrm{n}=$ $\mathrm{rk}(\mathrm{k}-2)$; when $\mathrm{c}=3 \mathrm{k}, \mathrm{n}=\mathrm{rk}(\mathrm{k}-3)$. In a plant breeding investigation, many of the new treatments may not be harvested or $\mathrm{n}$ may be less than the preceding numbers. Since only the replicated check treatments contribute to estimating the blocking parameters, whether or not a new treatment plot is harvested, does not affect the estimate of the error mean square or of the blocking parameters. Hence, the augmented designs have considerable flexibility concerning the inclusion of new treatment results in the final analysis.

The construction of ALSDs for $\mathrm{c}=2 \mathrm{k}$ checks is presented in the next section. The method is illustrated using three examples for $\mathrm{k}=4,5$, and 7 . When $\mathrm{c}=3 \mathrm{k}$ check treatments, the method of construction is illustrated using $k=13$. The number of replicates (complete blocks) is $\mathrm{r}=\mathrm{k}$. Fewer replicates may be used if the experimenter desires. Also, it is possible to construct additional replicates for ALSDs (See Federer,1995).

\section{ALSD CONSTRUCTION FOR $\mathrm{c}=\mathbf{2 k}$}

To construct an augmented lattice square design (ALSD), use is made of the results presented by Federer (1995). It was shown how to construct incomplete blocks of sizes 2 and 3 using a simple procedure called the row-column method. For $\mathrm{v}=2 \mathrm{k}$, entries 1 to $\mathrm{k}$ are placed on the main right diagonal of a $\mathrm{k}$ by $\mathrm{k}$ lattice. Entries $\mathrm{k}+1$ to $2 \mathrm{k}$ are placed on the right diagonal below the main right diagonal and are cyclically permuted to obtain the $\mathrm{r}=\mathrm{k}$ replicates.

For $\mathrm{k}=4$ rows and 4 columns, $\mathrm{c}=2 \mathrm{k}=8, \mathrm{r}=\mathrm{k}=4, \mathrm{n}=\mathrm{rk}(\mathrm{k}-2)=32$, and $\mathrm{x}$ denoting a new treatment, the unrandomized plan is:

\begin{tabular}{|c|c|c|c|c|c|c|c|c|c|c|c|c|c|}
\hline \multicolumn{3}{|c|}{ Replicate 1} & \multicolumn{3}{|c|}{ Replicate 2} & \multicolumn{4}{|c|}{ Replicate 3} & \multicolumn{4}{|c|}{ Replicate 4} \\
\hline $1 \mathrm{x}$ & $\mathrm{x}$ & 8 & $1 \mathrm{x}$ & $\mathrm{x}$ & 7 & 1 & & & 6 & & & & \\
\hline 2 & $\mathrm{x}$ & $\mathrm{X}$ & 82 & $\mathrm{x}$ & $\mathrm{x}$ & 7 & & & $\mathbf{X}$ & 6 & & & $\mathbf{X}$ \\
\hline 6 & 3 & $\lambda$ & 5 & 3 & $\mathrm{x}$ & & & & $\mathrm{x}$ & $\mathrm{x}$ & & & X \\
\hline $\mathrm{x}$ & 7 & 4 & $\mathrm{x} \quad \mathrm{x}$ & 6 & 4 & & & & 4 & $\mathrm{X}$ & & & 4 \\
\hline
\end{tabular}

For $\mathrm{k}=5$ rows and 5 columns, $\mathrm{c}=2 \mathrm{k}=10, \mathrm{r}=\mathrm{k}=5, \mathrm{n}=\mathrm{rk}(\mathrm{k}-2)=75$, and $\mathrm{x}$ denoting a new treatment, the unrandomized plan is:

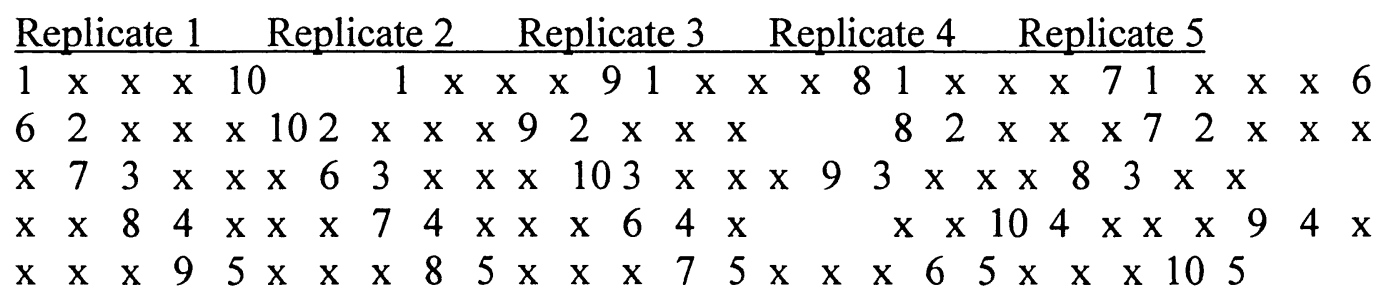

For $\mathrm{k}=7$ rows and 7 columns, $\mathrm{c}=2 \mathrm{k}=14, \mathrm{r}=\mathrm{k}=7, \mathrm{n}=\mathrm{rk}(\mathrm{k}-2)=245$, and $\mathrm{x}$ denoting a new treatment, the unrandomized plan is: 


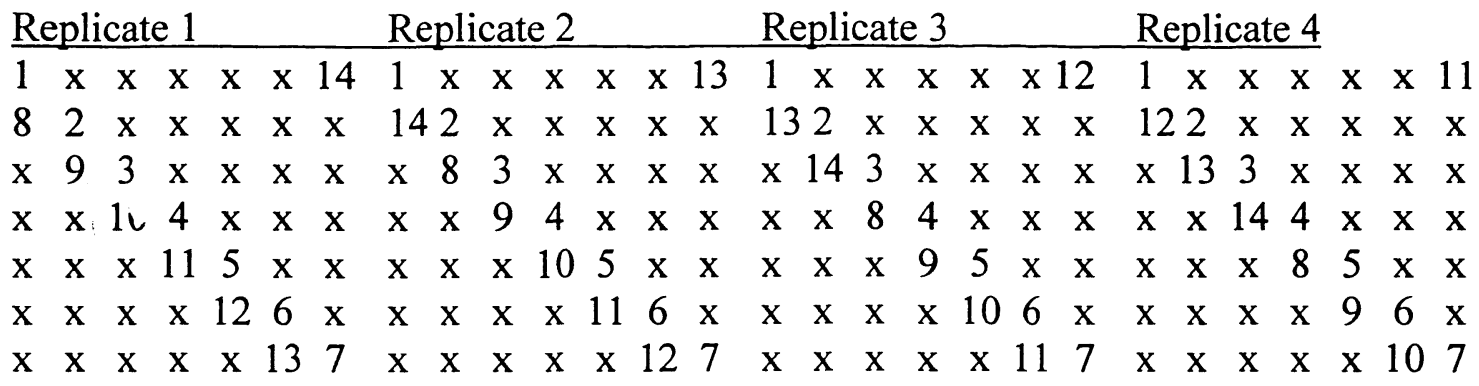

\begin{tabular}{|c|c|c|c|c|c|c|c|c|c|c|c|c|c|c|c|c|c|c|}
\hline Repli & cate & & & & & Repli & cat & e 6 & & & & & pli & cate & & & & \\
\hline $1 x$ & $\bar{x}$ & $\mathrm{X}$ & $\mathrm{X}$ & $x$ & 10 & $1 x$ & $\mathbf{x}$ & $\mathrm{X}$ & $\mathrm{X}$ & $\mathbf{X}$ & 9 & 1 & $\mathrm{X}$ & $\mathrm{x}$ & $\mathrm{x}$ & $\mathrm{X}$ & $\mathrm{X}$ & 8 \\
\hline 112 & $\mathrm{x}$ & $\mathrm{x}$ & $\mathrm{X}$ & $\mathrm{x}$ & $x$ & 102 & $\mathrm{x}$ & $\mathrm{x}$ & $\mathrm{X}$ & $\mathrm{x}$ & $\mathrm{x}$ & 9 & 2 & $\mathrm{x}$ & $\mathrm{x}$ & $\mathrm{x}$ & $x$ & $\mathrm{x}$ \\
\hline X 12 & 3 & $\mathrm{x}$ & $\mathrm{X}$ & $\mathrm{x}$ & $\mathrm{X}$ & X 11 & 3 & $\mathrm{x}$ & $\mathrm{X}$ & $\mathrm{x}$ & $\mathrm{X}$ & $\mathrm{x}$ & 10 & 3 & $\mathrm{x}$ & $\mathrm{x}$ & $\mathrm{X}$ & $\mathrm{X}$ \\
\hline$x \quad x$ & 13 & 4 & $\mathrm{X}$ & $\mathrm{x}$ & $x$ & $\mathrm{X} \quad \mathrm{X}$ & 12 & 4 & $\mathrm{X}$ & $\mathrm{x}$ & $\mathrm{X}$ & $\mathrm{x}$ & $\mathrm{X}$ & 11 & 4 & $\mathrm{x}$ & $\mathrm{x}$ & $\mathrm{x}$ \\
\hline $\mathrm{x} \quad \mathrm{x}$ & $\mathrm{x}$ & 14 & 5 & $\mathrm{x}$ & $\mathrm{x}$ & $\mathrm{X} \quad \mathrm{X}$ & $\mathrm{x}$ & 13 & 5 & $\mathrm{X}$ & $\mathbf{X}$ & $\mathrm{x}$ & $\mathrm{X}$ & $\mathbf{X}$ & 12 & 5 & $\mathrm{X}$ & $X$ \\
\hline$x \quad x$ & $\mathrm{x}$ & $\mathrm{x}$ & 8 & 6 & $x$ & $\mathrm{X} \quad \mathrm{X}$ & $\mathrm{x}$ & $\mathrm{x}$ & 14 & 6 & $\mathrm{X}$ & $\mathrm{X}$ & $\mathrm{X}$ & $\mathrm{x}$ & $\mathrm{X}$ & 13 & 6 & $\mathrm{X}$ \\
\hline $\mathrm{x} \quad \mathrm{x}$ & $\mathrm{x}$ & $\mathrm{x}$ & $\mathrm{x}$ & 9 & 7 & $\mathrm{X} \quad \mathrm{X}$ & $\mathrm{x}$ & $\mathrm{x}$ & $\mathrm{x}$ & 8 & 7 & $\mathrm{x}$ & $\mathrm{x}$ & $\mathrm{X}$ & $\mathrm{x}$ & $\mathrm{x}$ & 14 & 7 \\
\hline
\end{tabular}

In the above plans, treatments 1 to $\mathrm{k}$ do not appear together in any row or column.

Treatments 1 to $\mathrm{k}$ each appear once with each of the treatments $\mathrm{k}+1$ to $2 \mathrm{k}$ in rows and in columns. This is the best row and column association that can be achieved with this type of ALSD in rows and in columns. Also treatments $\mathrm{k}+1$ to $2 \mathrm{k}$ do not appear together in any row or any column. Hence, these ALSDs are optimal for these values of $c, k$, and $r$ as no better association scheme is possible.

The values of $\mathrm{n}$ given above are small compared to the usual numbers of new genotypes, treatments, being screened in standard plant breeding investigations. Some programs in sugarcane screen 30,000 new genotypes each year, which would require k to be 33 . Some programs on wheat regularly screen 8,000 new genotypes each year. An experimenter may wish to use fewer than $r=33$ replicates on some promising new treatments. Two, three, or s common checks could be included in all 33 replicates and three sets of $\mathrm{c}$ - s promising survivors from previous screening tests could be replicated 11 times each. Or, 11 sets of $\mathrm{c}-\mathrm{s}$ survivors could each be replicated three times.

The randomization procedure for an ALSD is to permute the rows and columns randomly in each of the $r$ replicates and to assign the numbers 1 to $2 \mathrm{k}$ to the check treatments at random. Since new treatments are untried and unknown, numbers 1 to $n$ may be assigned to them without randomization unless there is some grouping of the genotypes.

\section{ALSD CONSTRUCTION FOR $\mathrm{c}=3 \mathrm{k}$}

Use is made of the incomplete block designs of size three given by Federer (1995). Instead of placing the treatments in two diagonals, three diagonals are filled with treatments to make incomplete blocks of treatments in both rows and columns. 
Treatments 1 to $\mathrm{k}$ are placed on the main right diagonal of the $\mathrm{k}$ by $\mathrm{k}$ lattice in each of the $\mathrm{r}=\mathrm{k}$ replicates as in the previous section. Treatments $\mathrm{k}+1$ to $2 \mathrm{k}$ are placed on the right diagonal below the main right diagonal as in the previous section. To this point, the plans are identical to those constructed in the previous section. For treatments $2 \mathrm{k}+1$ to $3 \mathrm{k}$, the second right diagonal below the main right diagonal is used. The treatments need to be permuted as described by Federer (1995) in such a manner that no pair of treatments occurs together more than once in any row or column of the $r=k$ replicates. Doing this results in optimal ALSDs. The method is illustrated for $\mathrm{k}=\mathrm{r}=13, \mathrm{c}=3 \mathrm{k}=39$, and $\mathrm{n}=$ $\operatorname{rk}(\mathrm{k}-3)=1,690$ new treatments marked as $\mathrm{x}$.

\section{Replicate 1}

\begin{tabular}{lllllllllllllll}
\hline 1 & $\mathrm{x}$ & $\mathrm{x}$ & $\mathrm{x}$ & $\mathrm{x}$ & $\mathrm{x}$ & $\mathrm{x}$ & $\mathrm{x}$ & $\mathrm{x}$ & $\mathrm{x}$ & $\mathrm{x}$ & 38 & 26
\end{tabular}

$\begin{array}{lllllllllllllllll}142 & \mathrm{x} & \mathrm{x} & \mathrm{x} & \mathrm{x} & \mathrm{x} & \mathrm{x} & \mathrm{x} & \mathrm{x} & \mathrm{x} & \mathrm{x} & 39\end{array}$

$\begin{array}{llllllllllll}39 & 153 & \mathrm{x} & \mathrm{x} & \mathrm{x} & \mathrm{x} & \mathrm{x} & \mathrm{x} & \mathrm{x} & \mathrm{x} & \mathrm{x} & \mathrm{x}\end{array}$

$\begin{array}{llllllllllll}x & 27 & 164 & x & x & x & x & x & x & x & x & x\end{array}$

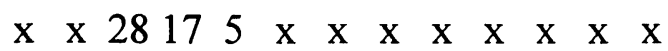

$\begin{array}{llllllllllllll}x & x & x & 29 & 18 & 6 & x & x & x & x & x & x & x\end{array}$

$\begin{array}{llllllllllllll}\mathrm{x} & \mathrm{x} & \mathrm{x} & \mathrm{x} & 30 & 19 & 7 & \mathrm{x} & \mathrm{x} & \mathrm{x} & \mathrm{x} & \mathrm{x} & \mathrm{x}\end{array}$

$\begin{array}{lllllllllllll}\mathrm{x} & \mathrm{x} & \mathrm{x} & \mathrm{x} & \mathrm{x} & 31 & 20 & 8 & \mathrm{x} & \mathrm{x} & \mathrm{x} & \mathrm{x} & \mathrm{x}\end{array}$

$\begin{array}{llllllllllllll}x & x & x & x & x & x & 32 & 21 & 9 & x & x & x & x\end{array}$

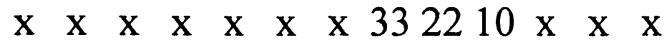

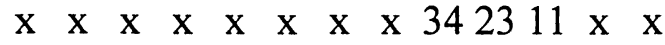

$\begin{array}{llllllllllllll}x & x & x & x & x & x & x & x & x & 35 & 24 & 12 & x\end{array}$

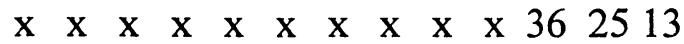

\section{Replicate 2}

$\begin{array}{lllllllllllll}1 & \mathrm{x} & \mathrm{x} & \mathrm{x} & \mathrm{x} & \mathrm{x} & \mathrm{x} & \mathrm{x} & \mathrm{x} & \mathrm{x} & \mathrm{x} & 36 & 25\end{array}$

$\begin{array}{llllllllllll}262 & x & x & x & x & x & x & x & x & x & x & 37\end{array}$

$\begin{array}{lllllllllllll}38 & 143 & x & x & x & x & x & x & x & x & x & x\end{array}$

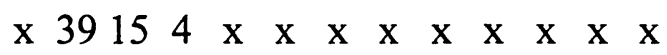

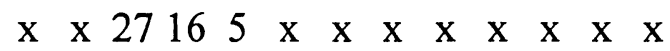

$\begin{array}{lllllllllllll}\mathrm{x} & \mathrm{x} & \mathrm{x} & 28 & 17 & 6 & \mathrm{x} & \mathrm{x} & \mathrm{x} & \mathrm{x} & \mathrm{x} & \mathrm{x} & \mathrm{x}\end{array}$

$\begin{array}{lllllllllllll}x & x & x & x & 29 & 18 & 7 & x & x & x & x & x & x\end{array}$

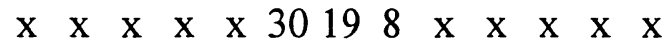

$\begin{array}{llllllllllllll}x & x & x & x & x & x & 31 & 20 & 9 & x & x & x & x\end{array}$

$\begin{array}{lllllllllllll}x & x & x & x & x & x & x & 32 & 21 & 10 & x & x & x\end{array}$

$\begin{array}{lllllllllllll}x & x & x & x & x & x & x & x & 33 & 22 & 11 & x & x\end{array}$

$\begin{array}{lllllllllllll}\mathrm{x} & \mathrm{x} & \mathrm{x} & \mathrm{x} & \mathrm{x} & \mathrm{x} & \mathrm{x} & \mathrm{x} & \mathrm{x} & 34 & 23 & 12 & \mathrm{x}\end{array}$

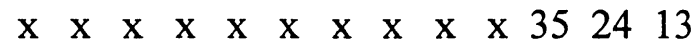

Replicate 3

$\begin{array}{llllllllllllll}1 & \mathrm{x} & \mathrm{x} & \mathrm{x} & \mathrm{x} & \mathrm{x} & \mathrm{x} & \mathrm{x} & \mathrm{x} & \mathrm{x} & \mathrm{x} & 34 & 24\end{array}$ $\begin{array}{llllllllllll}252 & \mathrm{x} & \mathrm{x} & \mathrm{x} & \mathrm{x} & \mathrm{x} & \mathrm{x} & \mathrm{x} & \mathrm{x} & \mathrm{x} & \mathrm{x} & 35\end{array}$ $\begin{array}{llllllllllll}36263 & \mathrm{x} & \mathrm{x} & \mathrm{x} & \mathrm{x} & \mathrm{x} & \mathrm{x} & \mathrm{x} & \mathrm{x} & \mathrm{x} & \mathrm{x}\end{array}$

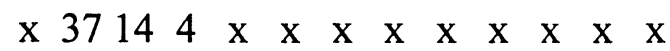

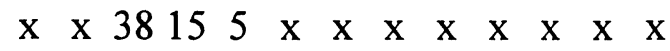
$\begin{array}{lllllllllllll}\mathrm{x} & \mathrm{x} & \mathrm{x} & 39 & 16 & 6 & \mathrm{x} & \mathrm{x} & \mathrm{x} & \mathrm{x} & \mathrm{x} & \mathrm{x} & \mathrm{x}\end{array}$ $\begin{array}{llllllllllllll}\mathrm{x} & \mathrm{x} & \mathrm{x} & \mathrm{x} & 27 & 17 & 7 & \mathrm{x} & \mathrm{x} & \mathrm{x} & \mathrm{x} & \mathrm{x} & \mathrm{x}\end{array}$ $\begin{array}{llllllllllllll}\mathrm{x} & \mathrm{x} & \mathrm{x} & \mathrm{x} & \mathrm{x} & 28 & 18 & 8 & \mathrm{x} & \mathrm{x} & \mathrm{x} & \mathrm{x} & \mathrm{x}\end{array}$ $\begin{array}{llllllllllllll}x & x & x & x & x & x & 29 & 19 & 9 & x & x & x & x\end{array}$ $\begin{array}{llllllllllllll}x & x & x & x & x & x & x & 30 & 20 & 10 & x & x & x\end{array}$

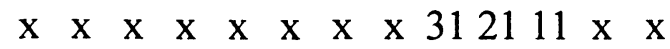

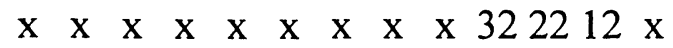
$\begin{array}{llllllllllllll}x & x & x & x & x & x & x & x & x & x & 33 & 23 & 13\end{array}$
Replicate 4

$\begin{array}{lllllllllllll}1 & \mathrm{x} & \mathrm{x} & \mathrm{x} & \mathrm{x} & \mathrm{x} & \mathrm{x} & \mathrm{x} & \mathrm{x} & \mathrm{x} & \mathrm{x} & 32 & 23\end{array}$

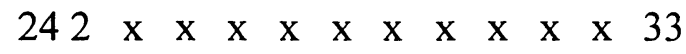
$\begin{array}{llllllllllll}34253 & \mathrm{x} & \mathrm{x} & \mathrm{x} & \mathrm{x} & \mathrm{x} & \mathrm{x} & \mathrm{x} & \mathrm{x} & \mathrm{x} & \mathrm{x}\end{array}$

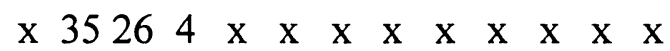

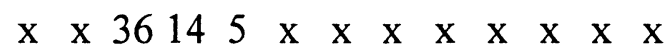
$\begin{array}{lllllllllllll}x & x & x & 37 & 15 & 6 & x & x & x & x & x & x & x\end{array}$ $\begin{array}{llllllllllllll}x & x & x & x & 38 & 16 & 7 & x & x & x & x & x & x\end{array}$ $\begin{array}{llllllllllllll}\mathrm{x} & \mathrm{x} & \mathrm{x} & \mathrm{x} & \mathrm{x} & 39 & 17 & 8 & \mathrm{x} & \mathrm{x} & \mathrm{x} & \mathrm{x} & \mathrm{x}\end{array}$ $\begin{array}{lllllllllllllll}\mathrm{x} & \mathrm{x} & \mathrm{x} & \mathrm{x} & \mathrm{x} & \mathrm{x} & 27 & 18 & 9 & \mathrm{x} & \mathrm{x} & \mathrm{x} & \mathrm{x}\end{array}$

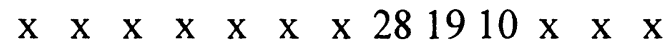

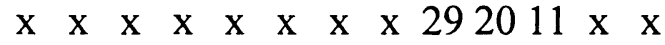

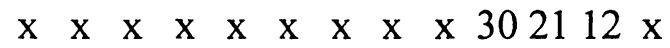

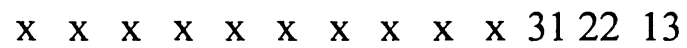


Replicate 5

$\begin{array}{lllllllllllll}1 & \mathrm{x} & \mathrm{x} & \mathrm{x} & \mathrm{x} & \mathrm{x} & \mathrm{x} & \mathrm{x} & \mathrm{x} & \mathrm{x} & \mathrm{x} & 30 & 22 \\ 23 & 2 & \mathrm{x} & \mathrm{x} & \mathrm{x} & \mathrm{x} & \mathrm{x} & \mathrm{x} & \mathrm{x} & \mathrm{x} & \mathrm{x} & \mathrm{x} & 31 \\ 32 & 24 & 3 & \mathrm{x} & \mathrm{x} & \mathrm{x} & \mathrm{x} & \mathrm{x} & \mathrm{x} & \mathrm{x} & \mathrm{x} & \mathrm{x} & \mathrm{x} \\ \mathrm{x} & 33 & 25 & 4 & \mathrm{x} & \mathrm{x} & \mathrm{x} & \mathrm{x} & \mathrm{x} & \mathrm{x} & \mathrm{x} & \mathrm{x} & \mathrm{x} \\ \mathrm{x} & \mathrm{x} & 3 & 26 & 5 & \mathrm{x} & \mathrm{x} & \mathrm{x} & \mathrm{x} & \mathrm{x} & \mathrm{x} & \mathrm{x} & \mathrm{x} \\ \mathrm{x} & \mathrm{x} & \mathrm{x} & 35 & 14 & 6 & \mathrm{x} & \mathrm{x} & \mathrm{x} & \mathrm{x} & \mathrm{x} & \mathrm{x} & \mathrm{x} \\ \mathrm{x} & \mathrm{x} & \mathrm{x} & \mathrm{x} & 36 & 15 & 7 & \mathrm{x} & \mathrm{x} & \mathrm{x} & \mathrm{x} & \mathrm{x} & \mathrm{x} \\ \mathrm{x} & \mathrm{x} & \mathrm{x} & \mathrm{x} & \mathrm{x} & 37 & 16 & 8 & \mathrm{x} & \mathrm{x} & \mathrm{x} & \mathrm{x} & \mathrm{x} \\ \mathrm{x} & \mathrm{x} & \mathrm{x} & \mathrm{x} & \mathrm{x} & \mathrm{x} & 38 & 17 & 9 & \mathrm{x} & \mathrm{x} & \mathrm{x} & \mathrm{x} \\ \mathrm{x} & \mathrm{x} & \mathrm{x} & \mathrm{x} & \mathrm{x} & \mathrm{x} & \mathrm{x} & 39 & 18 & 10 & \mathrm{x} & \mathrm{x} & \mathrm{x} \\ \mathrm{x} & \mathrm{x} & \mathrm{x} & \mathrm{x} & \mathrm{x} & \mathrm{x} & \mathrm{x} & \mathrm{x} & 27 & 19 & 11 & \mathrm{x} & \mathrm{x} \\ \mathrm{x} & \mathrm{x} & \mathrm{x} & \mathrm{x} & \mathrm{x} & \mathrm{x} & \mathrm{x} & \mathrm{x} & \mathrm{x} & 28 & 20 & 12 & \mathrm{x} \\ \mathrm{x} & \mathrm{x} & \mathrm{x} & \mathrm{x} & \mathrm{x} & \mathrm{x} & \mathrm{x} & \mathrm{x} & \mathrm{x} & \mathrm{x} & 29 & 21 & 13\end{array}$

Replicate 6

$\begin{array}{lllllllllllll}1 & \mathrm{x} & \mathrm{x} & \mathrm{x} & \mathrm{x} & \mathrm{x} & \mathrm{x} & \mathrm{x} & \mathrm{x} & \mathrm{x} & \mathrm{x} & 28 & 21 \\ 22 & 2 & \mathrm{x} & \mathrm{x} & \mathrm{x} & \mathrm{x} & \mathrm{x} & \mathrm{x} & \mathrm{x} & \mathrm{x} & \mathrm{x} & \mathrm{x} & 29 \\ 30 & 23 & 3 & \mathrm{x} & \mathrm{x} & \mathrm{x} & \mathrm{x} & \mathrm{x} & \mathrm{x} & \mathrm{x} & \mathrm{x} & \mathrm{x} & \mathrm{x} \\ \mathrm{x} & 31 & 24 & 4 & \mathrm{x} & \mathrm{x} & \mathrm{x} & \mathrm{x} & \mathrm{x} & \mathrm{x} & \mathrm{x} & \mathrm{x} & \mathrm{x} \\ \mathrm{x} & \mathrm{x} & 32 & 25 & 5 & \mathrm{x} & \mathrm{x} & \mathrm{x} & \mathrm{x} & \mathrm{x} & \mathrm{x} & \mathrm{x} & \mathrm{x} \\ \mathrm{x} & \mathrm{x} & \mathrm{x} & 33 & 26 & 6 & \mathrm{x} & \mathrm{x} & \mathrm{x} & \mathrm{x} & \mathrm{x} & \mathrm{x} & \mathrm{x} \\ \mathrm{x} & \mathrm{x} & \mathrm{x} & \mathrm{x} & 34 & 14 & 7 & \mathrm{x} & \mathrm{x} & \mathrm{x} & \mathrm{x} & \mathrm{x} & \mathrm{x} \\ \mathrm{x} & \mathrm{x} & \mathrm{x} & \mathrm{x} & \mathrm{x} & 35 & 15 & 8 & \mathrm{x} & \mathrm{x} & \mathrm{x} & \mathrm{x} & \mathrm{x} \\ \mathrm{x} & \mathrm{x} & \mathrm{x} & \mathrm{x} & \mathrm{x} & \mathrm{x} & 36 & 18 & 9 & \mathrm{x} & \mathrm{x} & \mathrm{x} & \mathrm{x} \\ \mathrm{x} & \mathrm{x} & \mathrm{x} & \mathrm{x} & \mathrm{x} & \mathrm{x} & \mathrm{x} & 37 & 19 & 10 & \mathrm{x} & \mathrm{x} & \mathrm{x} \\ \mathrm{x} & \mathrm{x} & \mathrm{x} & \mathrm{x} & \mathrm{x} & \mathrm{x} & \mathrm{x} & \mathrm{x} & 38 & 20 & 11 & \mathrm{x} & \mathrm{x} \\ \mathrm{x} & \mathrm{x} & \mathrm{x} & \mathrm{x} & \mathrm{x} & \mathrm{x} & \mathrm{x} & \mathrm{x} & \mathrm{x} & 39 & 21 & 12 & \mathrm{x} \\ \mathrm{x} & \mathrm{x} & \mathrm{x} & \mathrm{x} & \mathrm{x} & \mathrm{x} & \mathrm{x} & \mathrm{x} & \mathrm{x} & \mathrm{x} & 27 & 22 & 13\end{array}$

Replicate 7

Replicate 8

$\begin{array}{llllllllllllll}1 & x & x & x & x & x & x & x & x & x & x & 39 & 20\end{array}$ $\begin{array}{llllllllllllll}1 & \mathrm{x} & \mathrm{x} & \mathrm{x} & \mathrm{x} & \mathrm{x} & \mathrm{x} & \mathrm{x} & \mathrm{x} & \mathrm{x} & \mathrm{x} & 37 & & \end{array}$ $\begin{array}{lllllllllllll}212 & x & x & x & x & x & x & x & x & x & x & 27\end{array}$

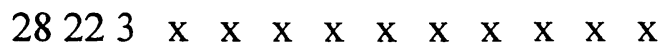

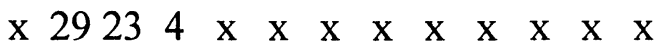

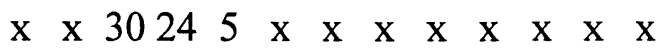

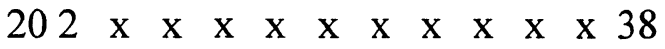

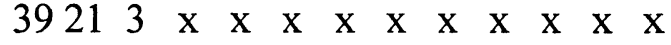

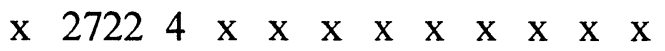

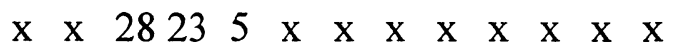

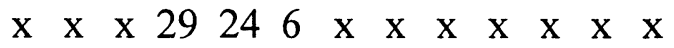
$\begin{array}{lllllllllllll}x & x & x & x & 30 & 25 & 7 & x & x & x & x & x & x\end{array}$ $\begin{array}{llllllllllllll}x & x & x & x & x & 31 & 26 & 8 & x & x & x & x & x\end{array}$

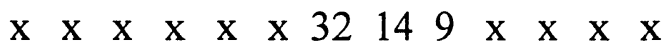

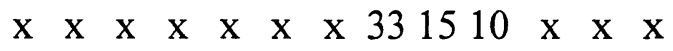

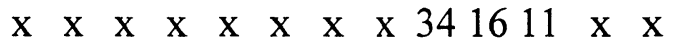
$\begin{array}{llllllllllllll}\mathrm{x} & \mathrm{x} & \mathrm{x} & \mathrm{x} & \mathrm{x} & \mathrm{x} & \mathrm{x} & \mathrm{x} & \mathrm{x} & 35 & 17 & 12 & \mathrm{x}\end{array}$

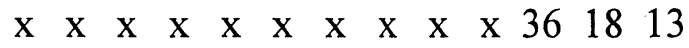

Replicate 9

$\begin{array}{llllllllllllllllllllllllll}1 & \mathrm{x} & \mathrm{x} & \mathrm{x} & \mathrm{x} & \mathrm{x} & \mathrm{x} & \mathrm{x} & \mathrm{x} & \mathrm{x} & \mathrm{x} & 35 & 18 & 1 & \mathrm{x} & \mathrm{x} & \mathrm{x} & \mathrm{x} & \mathrm{x} & \mathrm{x} & \mathrm{x} & \mathrm{x} & \mathrm{x} & \mathrm{x} & 33 & 17\end{array}$

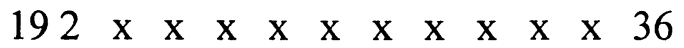

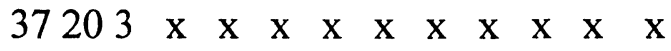
$\begin{array}{lllllllllllll}18 & 2 & \mathrm{x} & \mathrm{x} & \mathrm{x} & \mathrm{x} & \mathrm{x} & \mathrm{x} & \mathrm{x} & \mathrm{x} & \mathrm{x} & \mathrm{x} & 34\end{array}$

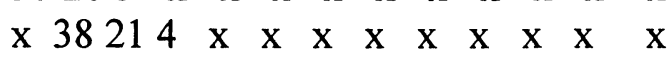
$\begin{array}{llllllllllll}35 & 193 & \mathrm{x} & \mathrm{x} & \mathrm{x} & \mathrm{x} & \mathrm{x} & \mathrm{x} & \mathrm{x} & \mathrm{x} & \mathrm{x} & \mathrm{x}\end{array}$ $\begin{array}{llllllllllllllllllllllllll}x & x & 39 & 22 & 5 & x & x & x & x & x & x & x & x\end{array}$

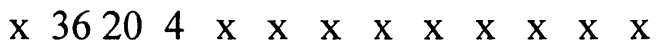

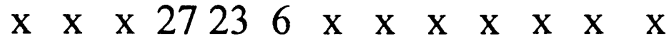

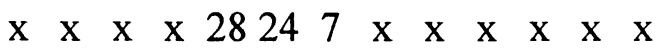

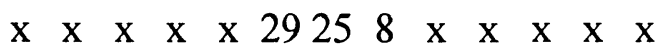

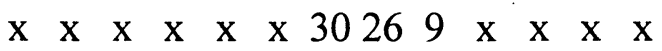
$\begin{array}{lllllllllllll}\mathrm{x} & \mathrm{x} & \mathrm{x} & \mathrm{x} & \mathrm{x} & \mathrm{x} & \mathrm{x} & 31 & 14 & 10 & \mathrm{x} & \mathrm{x} & \mathrm{x}\end{array}$ $\begin{array}{lllllllllllll}x & x & x & x & x & x & x & x & 32 & 15 & 11 & x & x\end{array}$ $\begin{array}{lllllllllllllllllllllll}x & x & x & x & x & x & x & x & x & 33 & 16 & 12 & x\end{array}$

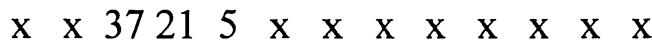

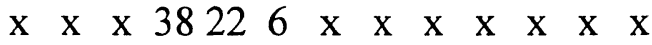

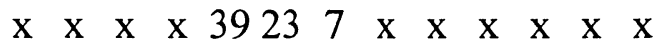

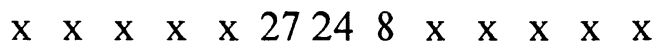
$\begin{array}{lllllllllllll}x & x & x & x & x & x & 28 & 25 & 9 & x & x & x & x\end{array}$

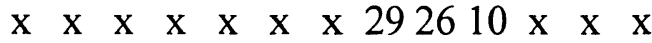

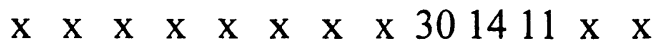

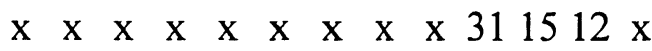

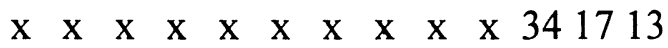

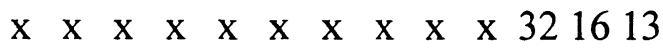


Replicate $11 \quad$ Replicate 12

$\begin{array}{lllllllllllllllllllllllllll}1 & \mathrm{x} & \mathrm{x} & \mathrm{x} & \mathrm{x} & \mathrm{x} & \mathrm{x} & \mathrm{x} & \mathrm{x} & \mathrm{x} & \mathrm{x} & 31 & 16 & 1 & \mathrm{x} & \mathrm{x} & \mathrm{x} & \mathrm{x} & \mathrm{x} & \mathrm{x} & \mathrm{x} & \mathrm{x} & \mathrm{x} & \mathrm{x} & 29 & 15 \\ 17 & 2 & \mathrm{x} & \mathrm{x} & \mathrm{x} & \mathrm{x} & \mathrm{x} & \mathrm{x} & \mathrm{x} & \mathrm{x} & \mathrm{x} & \mathrm{x} & 32 & 16 & 2 & \mathrm{x} & \mathrm{x} & \mathrm{x} & \mathrm{x} & \mathrm{x} & \mathrm{x} & \mathrm{x} & \mathrm{x} & \mathrm{x} & \mathrm{x} & 30 \\ 33 & 18 & 3 & \mathrm{x} & \mathrm{x} & \mathrm{x} & \mathrm{x} & \mathrm{x} & \mathrm{x} & \mathrm{x} & \mathrm{x} & \mathrm{x} & \mathrm{x} & 31 & 17 & 3 & \mathrm{x} & \mathrm{x} & \mathrm{x} & \mathrm{x} & \mathrm{x} & \mathrm{x} & \mathrm{x} & \mathrm{x} & \mathrm{x} & \mathrm{x} \\ \mathrm{x} & 34 & 19 & 4 & \mathrm{x} & \mathrm{x} & \mathrm{x} & \mathrm{x} & \mathrm{x} & \mathrm{x} & \mathrm{x} & \mathrm{x} & \mathrm{x} & \mathrm{x} & 32 & 18 & 4 & \mathrm{x} & \mathrm{x} & \mathrm{x} & \mathrm{x} & \mathrm{x} & \mathrm{x} & \mathrm{x} & \mathrm{x} & \mathrm{x} \\ \mathrm{x} & \mathrm{x} & 35 & 20 & 5 & \mathrm{x} & \mathrm{x} & \mathrm{x} & \mathrm{x} & \mathrm{x} & \mathrm{x} & \mathrm{x} & \mathrm{x} & \mathrm{x} & \mathrm{x} & 33 & 19 & 5 & \mathrm{x} & \mathrm{x} & \mathrm{x} & \mathrm{x} & \mathrm{x} & \mathrm{x} & \mathrm{x} & \mathrm{x} \\ \mathrm{x} & \mathrm{x} & \mathrm{x} & 36 & 21 & 6 & \mathrm{x} & \mathrm{x} & \mathrm{x} & \mathrm{x} & \mathrm{x} & \mathrm{x} & \mathrm{x} & \mathrm{x} & \mathrm{x} & \mathrm{x} & 34 & 20 & 6 & \mathrm{x} & \mathrm{x} & \mathrm{x} & \mathrm{x} & \mathrm{x} & \mathrm{x} & \mathrm{x} \\ \mathrm{x} & \mathrm{x} & \mathrm{x} & \mathrm{x} & 37 & 22 & 7 & \mathrm{x} & \mathrm{x} & \mathrm{x} & \mathrm{x} & \mathrm{x} & \mathrm{x} & \mathrm{x} & \mathrm{x} & \mathrm{x} & \mathrm{x} & 35 & 21 & 7 & \mathrm{x} & \mathrm{x} & \mathrm{x} & \mathrm{x} & \mathrm{x} & \mathrm{x} \\ \mathrm{x} & \mathrm{x} & \mathrm{x} & \mathrm{x} & \mathrm{x} & 38 & 23 & 8 & \mathrm{x} & \mathrm{x} & \mathrm{x} & \mathrm{x} & \mathrm{x} & \mathrm{x} & \mathrm{x} & \mathrm{x} & \mathrm{x} & \mathrm{x} & 36 & 22 & 8 & \mathrm{x} & \mathrm{x} & \mathrm{x} & \mathrm{x} & \mathrm{x} \\ \mathrm{x} & \mathrm{x} & \mathrm{x} & \mathrm{x} & \mathrm{x} & \mathrm{x} & 39 & 24 & 9 & \mathrm{x} & \mathrm{x} & \mathrm{x} & \mathrm{x} & \mathrm{x} & \mathrm{x} & \mathrm{x} & \mathrm{x} & \mathrm{x} & \mathrm{x} & 37 & 23 & 9 & \mathrm{x} & \mathrm{x} & \mathrm{x} & \mathrm{x} \\ \mathrm{x} & \mathrm{x} & \mathrm{x} & \mathrm{x} & \mathrm{x} & \mathrm{x} & \mathrm{x} & 27 & 25 & 10 & \mathrm{x} & \mathrm{x} & \mathrm{x} & \mathrm{x} & \mathrm{x} & \mathrm{x} & \mathrm{x} & \mathrm{x} & \mathrm{x} & \mathrm{x} & 38 & 24 & 10 & \mathrm{x} & \mathrm{x} & \mathrm{x} \\ \mathrm{x} & \mathrm{x} & \mathrm{x} & \mathrm{x} & \mathrm{x} & \mathrm{x} & \mathrm{x} & \mathrm{x} & 28 & 26 & 11 & \mathrm{x} & \mathrm{x} & \mathrm{x} & \mathrm{x} & \mathrm{x} & \mathrm{x} & \mathrm{x} & \mathrm{x} & \mathrm{x} & \mathrm{x} & 39 & 25 & 11 & \mathrm{x} & \mathrm{x} \\ \mathrm{x} & \mathrm{x} & \mathrm{x} & \mathrm{x} & \mathrm{x} & \mathrm{x} & \mathrm{x} & \mathrm{x} & \mathrm{x} & 29 & 14 & 12 & \mathrm{x} & \mathrm{x} & \mathrm{x} & \mathrm{x} & \mathrm{x} & \mathrm{x} & \mathrm{x} & \mathrm{x} & \mathrm{x} & \mathrm{x} & 27 & 26 & 12 & \mathrm{x} \\ \mathrm{x} & \mathrm{x} & \mathrm{x} & \mathrm{x} & \mathrm{x} & \mathrm{x} & \mathrm{x} & \mathrm{x} & \mathrm{x} & \mathrm{x} & 30 & 15 & 13 & \mathrm{x} & \mathrm{x} & \mathrm{x} & \mathrm{x} & \mathrm{x} & \mathrm{x} & \mathrm{x} & \mathrm{x} & \mathrm{x} & \mathrm{x} & 28 & 14 & 13\end{array}$

Replicate 13

$\begin{array}{lllllllllllll}1 & \mathrm{x} & \mathrm{x} & \mathrm{x} & \mathrm{x} & \mathrm{x} & \mathrm{x} & \mathrm{x} & \mathrm{x} & \mathrm{x} & \mathrm{x} & 27 & 14 \\ 15 & 2 & \mathrm{x} & \mathrm{x} & \mathrm{x} & \mathrm{x} & \mathrm{x} & \mathrm{x} & \mathrm{x} & \mathrm{x} & \mathrm{x} & \mathrm{x} & 28 \\ 29 & 16 & 3 & \mathrm{x} & \mathrm{x} & \mathrm{x} & \mathrm{x} & \mathrm{x} & \mathrm{x} & \mathrm{x} & \mathrm{x} & \mathrm{x} & \mathrm{x} \\ \mathrm{x} & 30 & 17 & 4 & \mathrm{x} & \mathrm{x} & \mathrm{x} & \mathrm{x} & \mathrm{x} & \mathrm{x} & \mathrm{x} & \mathrm{x} & \mathrm{x} \\ \mathrm{x} & \mathrm{x} & 31 & 18 & 5 & \mathrm{x} & \mathrm{x} & \mathrm{x} & \mathrm{x} & \mathrm{x} & \mathrm{x} & \mathrm{x} & \mathrm{x} \\ \mathrm{x} & \mathrm{x} & \mathrm{x} & 32 & 19 & 6 & \mathrm{x} & \mathrm{x} & \mathrm{x} & \mathrm{x} & \mathrm{x} & \mathrm{x} & \mathrm{x} \\ \mathrm{x} & \mathrm{x} & \mathrm{x} & \mathrm{x} & 33 & 20 & 7 & \mathrm{x} & \mathrm{x} & \mathrm{x} & \mathrm{x} & \mathrm{x} & \mathrm{x} \\ \mathrm{x} & \mathrm{x} & \mathrm{x} & \mathrm{x} & \mathrm{x} & 34 & 21 & 8 & \mathrm{x} & \mathrm{x} & \mathrm{x} & \mathrm{x} & \mathrm{x} \\ \mathrm{x} & \mathrm{x} & \mathrm{x} & \mathrm{x} & \mathrm{x} & \mathrm{x} & 35 & 22 & 9 & \mathrm{x} & \mathrm{x} & \mathrm{x} & \mathrm{x} \\ \mathrm{x} & \mathrm{x} & \mathrm{x} & \mathrm{x} & \mathrm{x} & \mathrm{x} & \mathrm{x} & 36 & 23 & 10 & \mathrm{x} & \mathrm{x} & \mathrm{x} \\ \mathrm{x} & \mathrm{x} & \mathrm{x} & \mathrm{x} & \mathrm{x} & \mathrm{x} & \mathrm{x} & \mathrm{x} & 37 & 24 & 11 & \mathrm{x} & \mathrm{x} \\ \mathrm{x} & \mathrm{x} & \mathrm{x} & \mathrm{x} & \mathrm{x} & \mathrm{x} & \mathrm{x} & \mathrm{x} & \mathrm{x} & 38 & 25 & 12 & \mathrm{x} \\ \mathrm{x} & \mathrm{x} & \mathrm{x} & \mathrm{x} & \mathrm{x} & \mathrm{x} & \mathrm{x} & \mathrm{x} & \mathrm{x} & \mathrm{x} & 39 & 26 & 13\end{array}$

A possibly better design would be to use the fourth diagonal down instead of the third. If it desired to use say the first third and fifth diagonals, one would need to determine if the desired row and column contrasts were estimable.

\section{STATISTICAL ANALYSES FOR ALSDs}

An analysis of variance for partitioning the degrees of freedom in an ALSD is given below for various values of $\mathrm{k}$. Using only $\mathrm{c}=2 \mathrm{k}$ checks, it is not possible to obtain solutions for all row and column effects. Hence some function of the row and column effects will be required. We shall use polynomial regression functions of the ordering of rows and columns. A partitioning of the degrees of freedom for values of $\mathrm{k}=3,4,5,6$, $7,8,9,10$ and 11 is: 


\begin{tabular}{|c|c|c|c|c|c|c|c|c|c|}
\hline \multirow{2}{*}{ Source of variation } & \multicolumn{2}{|c|}{ Value of $\mathrm{k}$} & \multirow[b]{2}{*}{5} & \multirow[b]{2}{*}{6} & \multirow[b]{2}{*}{7} & \multirow[b]{2}{*}{8} & \multirow[b]{2}{*}{9} & \multirow[b]{2}{*}{10} & \multirow[b]{2}{*}{11} \\
\hline & 3 & 4 & & & & & & & \\
\hline Total $=\mathrm{k}^{3}$ & 27 & 64 & 125 & 216 & 343 & 512 & 729 & 1000 & 1331 \\
\hline Correction for mean & 1 & 1 & 1 & 1 & 1 & 1 & 1 & 1 & 1 \\
\hline Replicate $=\mathrm{k}-1$ & 2 & 3 & 4 & 5 & 6 & 7 & 8 & 9 & 10 \\
\hline Entri is $=2 k+k^{2}(k-2)$ & 14 & 39 & 84 & 155 & 258 & 399 & 584 & 819 & 1110 \\
\hline Check & 5 & 7 & 9 & 11 & 13 & 15 & 17 & 19 & 21 \\
\hline Check vs. new & 1 & 1 & 1 & 1 & 1 & 1 & 1 & 1 & 1 \\
\hline New & 8 & 31 & 74 & 143 & 244 & 383 & 566 & 799 & 1088 \\
\hline Check by replicate & 10 & 21 & 36 & 55 & 78 & 105 & 136 & 171 & 210 \\
\hline Row linear within rep. & & 4 & 5 & 6 & 7 & 8 & 9 & 10 & 11 \\
\hline Column linear wn rep. & & 4 & 5 & 6 & 7 & 8 & 9 & 10 & 11 \\
\hline Row by col lin wn rep & & 4 & 5 & 6 & 7 & 8 & 9 & 10 & 11 \\
\hline Quadratic row wn. rep & & - & 5 & 6 & 7 & 8 & 9 & 10 & 11 \\
\hline Quadratic col wn rep & - & - & - & 6 & 7 & 8 & 9 & 10 & 11 \\
\hline Quad row by col wn re & & - & - & 6 & 7 & 8 & 9 & 10 & 11 \\
\hline Cubic row within rep. & & - & - & - & 7 & 8 & 9 & 10 & 11 \\
\hline Cubic col. wn. rep. & - & - & - & - & 7 & 8 & 9 & 10 & 11 \\
\hline Residual & 4 & 9 & 16 & 25 & 22 & 41 & 64 & 91 & 122 \\
\hline
\end{tabular}

As can be seen from the above, higher degree polynomials may be used to account for the spatial variation in ALSDs for the larger values of $k$.

\section{A NUMERICAL EXAMPLE OF AN AUGMENTED LATTICE SQUARE DESIGN}

A numerical example of an augmented lattice square design with $\mathrm{c}=8, \mathrm{k}=\mathrm{r}=3$, and $n=6$ new treatments is given below. Rep is replicate and col is column. The checks are numbered $7,8,9,10,11,12,13$, and 14; the new treatments are numbered from 1 to 6. The symbol for treatment is trt. This numbering system is used as the SAS code puts the highest numbered treatment effect equal to zero; this convention subtracts the highest numbered effect solution from all other treatment effects.

\begin{tabular}{lllll} 
rep & row & col & trt & Yield \\
\hline 1 & 1 & 1 & 10 & 15 \\
1 & 1 & 2 & 1 & 19 \\
1 & 1 & 3 & 15 & 25 \\
1 & 2 & 1 & 13 & 10 \\
1 & 2 & 2 & 11 & 10 \\
1 & 2 & 3 & 2 & 19 \\
1 & 3 & 1 & 3 & 19 \\
1 & 3 & 2 & 14 & 10 \\
1 & 3 & 3 & 12 & 10 \\
2 & 1 & 1 & 10 & 25 \\
2 & 1 & 2 & 4 & 10 \\
2 & 1 & 3 & 13 & 20 \\
2 & 2 & 1 & 14 & 10 \\
2 & 2 & 2 & 11 & 10 \\
2 & 2 & 3 & 5 & 10 \\
2 & 3 & 1 & 6 & 10 \\
2 & 3 & 2 & 15 & 20
\end{tabular}




$\begin{array}{lllll}2 & 3 & 3 & 12 & 10 \\ 3 & 1 & 1 & 10 & 20 \\ 3 & 1 & 2 & 7 & 10 \\ 3 & 1 & 3 & 14 & 10 \\ 3 & 2 & 1 & 15 & 25 \\ 3 & 2 & 2 & 11 & 15 \\ 3 & 2 & 3 & 8 & 22 \\ 3 & 3 & 1 & 9 & 24 \\ 3 & 3 & 2 & 13 & 10 \\ 3 & 3 & 3 & 12 & 10 ;\end{array}$

\section{A SAS CODE FOR ANALYZING THE DATA FROM ALSDS}

There is insufficient data to obtain solutions for all row and column effects in the above ALSD. Hence, some function of row and column effects will be needed. Trend analysis makes use of polynomial regression functions. For this small value of $k$, it will be possible to use only a linear trend for rows and column. The interaction of these regressions can be used but one degree of freedom is completely confounded with other contrasts, i.e., the sum of squares is associated with two instead of three degrees of freedom in the SAS/GLM analysis. This SAS program is for an augmented lattice square design with $2 \mathrm{k}$ and $3 \mathrm{k}$ checks for $\mathrm{k}=3$. The filename of the data set is auglsd6.dat. This program may also be used for analyses of augmented incomplete block, augmented row column, and augmented randomized complete blocks. options $1 \mathrm{~s}=76$;

proc iml;

opn3 $=\operatorname{orpol}(1: 3,2) ; /$ Change when $k$ is different from 3. *

opn $3[, 1]=(1: 3)^{\prime} ;$

op3 =opn3; print op3;

create opn3 from opn3 [colname $=\{$ 'COL' 'C1' 'C2' $\}$ ]; append from opn3;

close opn3; run;

opn4 $=$ orpol $(1: 3,2) ; /$ Change for other values of $\mathrm{k}$. */

opn $4[, 1]=(1: 3)$;

op4 =opn 4 ; print op4;

create opn4 from opn4 [colname $=\{$ 'ROW' 'R1' 'R2' $\}$ ]; append from opn4; close opn4; run;

data auglsd6;

infile 'auglsd6.dat';

input rep row col trt yield;

/* The following two lines divides the checks and new into two groups. The new treatments are usually taken to be random effects and the checks as fixed effects. */

if (trt>9) then new $=0$; else new $=1$;

if (new) then $\operatorname{trtn}=999$; else trtn = trt;

data augbig; set auglsd6;

$i d x=n$; run;

proc sort $\overline{\text { data }}=$ augbig;

by COL; run; data augbig; merge augbig opn3; by COL; run;

proc sort data = augbig;

by ROW; run; data augbig; merge augbig opn4; by ROW; run;

proc sort data = augbig; by idx; run;

proc glm data = augbig;

class row col trt trtn rep;

model yield $=$ rep trt $\mathrm{Cl}$ (rep) $\mathrm{R} 1$ (rep);

lsmeans trt/out = lsmeans noprint;

run; 


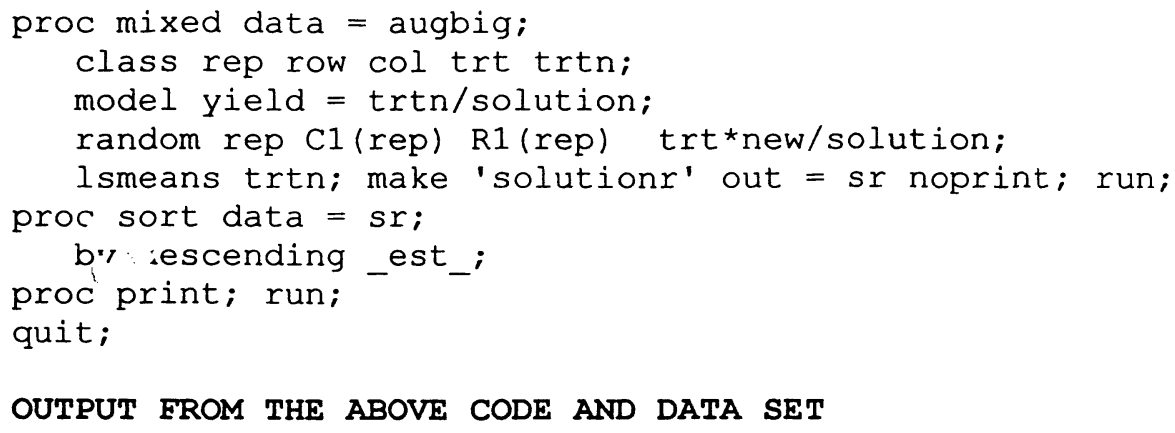

\section{OUTPUT FROM THE ABOVE CODE AND DATA SET}

An edited version of the output of the above code is given below. The code requested both the linear and quadratic coefficients. In standardized form these are:

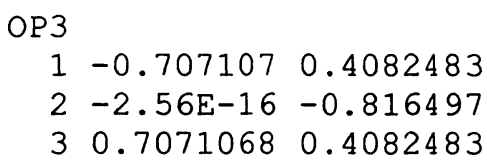

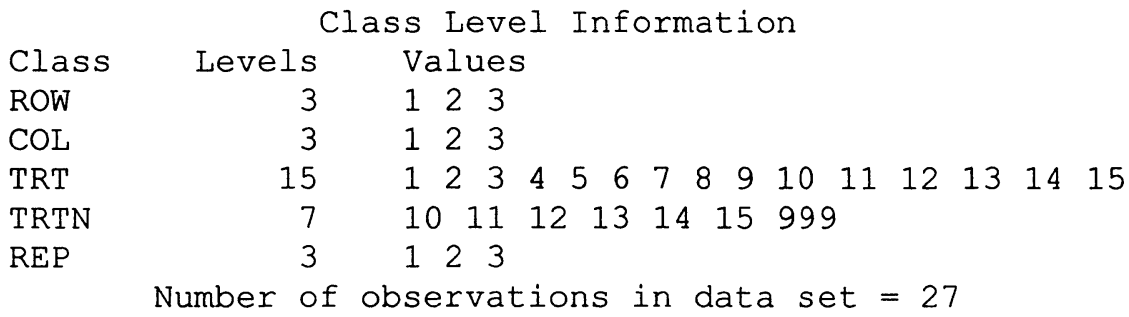

Dependent Variable: YIELD

\begin{tabular}{lrrrrr} 
& \multicolumn{4}{c}{ Sum of } & \multicolumn{2}{c}{ Mean } \\
Source & DF & Squares & Square & F Value & Pr $>$ F \\
Model & 22 & 874.888889 & 39.767677 & 5.73 & 0.0509 \\
Error & 4 & 27.777778 & 6.944444 & \\
Corrected Total & 26 & 902.666667 & & \\
& & & & & \\
& R-Square & C.V. & Root MSE & YIELD Mean \\
& 0.969227 & 17.43903 & 2.63523 & 15.1111
\end{tabular}

Dependent Variable: YIELD

$\begin{array}{lrrrrr}\text { Source } & \text { DE } & \text { Type I SS } & \text { Mean Square } & \text { F Value } & \text { Pr }>\text { F } \\ \text { REP } & 2 & 24.666667 & 12.333333 & 1.78 & 0.2805 \\ \text { TRT } & 14 & 747.444444 & 53.388889 & 7.69 & 0.0311 \\ \text { C1 (REP) } & 3 & 40.740741 & 13.580247 & 1.96 & 0.2627 \\ \text { R1 (REP) } & 3 & 62.037037 & 20.679012 & 2.98 & 0.1596 \\ & & & & & \\ \text { Source } & \text { DF } & \text { Type III SS } & \text { Mean Square } & \text { F Value } & \text { Pr }>\text { F } \\ \text { REP } & 2 & 19.44444 & 9.722222 & 1.40 & 0.3460 \\ \text { TRT } & 14 & 601.055556 & 42.932540 & 6.18 & 0.0457 \\ \text { C1 (REP) } & 3 & 7.870370 & 2.623457 & 0.38 & 0.7750 \\ \text { R1 (REP) } & 3 & 62.037037 & 20.679012 & 2.98 & 0.1596\end{array}$


The MIXED Procedure

REML Estimation Iteration History

$\begin{array}{rrrr}\text { Iteration } & \text { Evaluations } & \text { Objective } & \text { Criterion } \\ 0 & 1 & 89.72163038 & \\ 1 & 2 & 86.42847381 & 0.00842605 \\ 2 & 1 & 85.95599350 & 0.00385965 \\ 3 & 1 & 85.75053403 & 0.00100432 \\ 4 & 1 & 85.70023437 & 0.00010553 \\ 5 & 1 & 85.69537224 & 0.00000164 \\ 6 & 1 & 85.69530120 & 0.00000000\end{array}$

Convergence criteria met.

Covariance Parameter Estimates (REML)

Cov Parm Estimate

REP $\quad 0.00000000$

C1 (REP) $\quad 0.00000000$

R1 (REP) $\quad 12.34395756$

NEW*TRT $\quad 32.96359302$

Residual $\quad 7.11073420$

Model Fitting Information for YIELD

Description

Value

Observations

27.0000

Model Fitting Information for YIELD

Value

Res Log Likelihood $\quad-61.2264$

Akaike's Information Criterion -66.2264

Schwarz's Bayesian Criterion $\quad-68.7158$

-2 Res Log Likelihood 122.4528

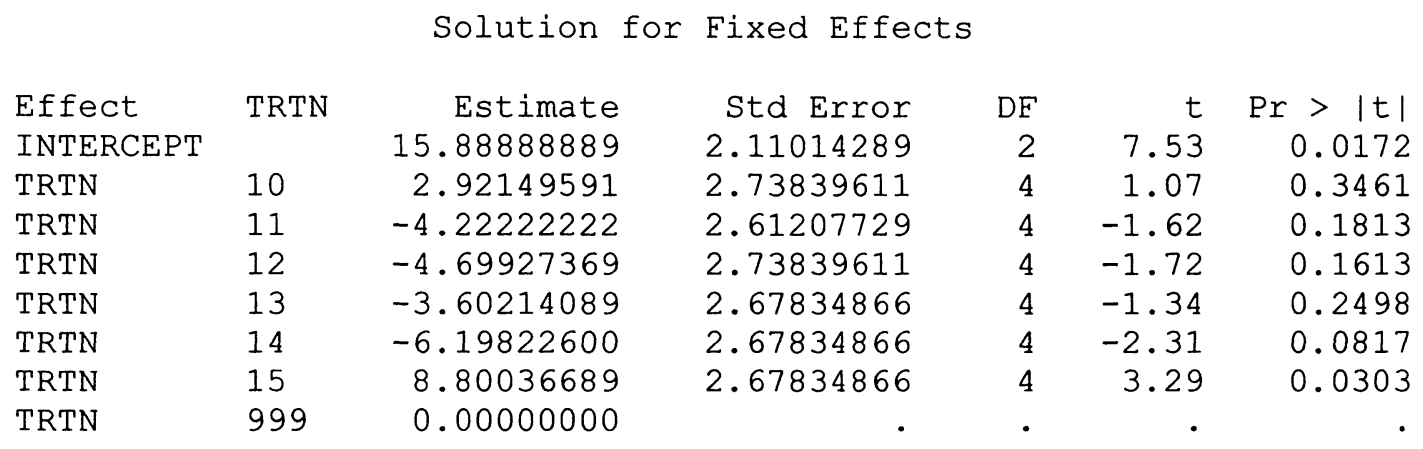

/* The intercept plus the effect solution is the mean given below. */

Tests of Fixed Effects

$\begin{array}{lrrrr}\text { Source } & \text { NDF } & \text { DDF } & \text { Type III F } & \text { Pr > F } \\ \text { TRTN } & 6 & 4 & 9.56 & 0.0234\end{array}$

Effect TRTN LSMEAN $\begin{array}{lrrr}\text { Std Error } & \text { DF } & \operatorname{Pr}>|t|\end{array}$




\begin{tabular}{|c|c|c|c|c|c|c|c|c|}
\hline & TRTN & 10 & & .81038480 & 1.74531093 & 4 & 10.78 & 0.0004 \\
\hline & TRTN & 11 & & .66666667 & 1.53955992 & 4 & 7.58 & 0.0016 \\
\hline & TRTN & 12 & & .18961520 & 1.74531093 & 4 & 6.41 & 0.0030 \\
\hline & TRTN & 13 & & .28674800 & 1.64949948 & 4 & 7.45 & 0.0017 \\
\hline & TRTN & 14 & & .69066288 & 1.64949948 & 4 & 5.87 & 0.0042 \\
\hline & TRTN & 15 & & .68925578 & 1.64949948 & 4 & 14.97 & 0.0001 \\
\hline & - RTN & 999 & & .88888889 & 2.11014289 & 4 & 7.53 & 0.0017 \\
\hline OBS & EFFECT & REP & TRT & EST & SEPRED & $\mathrm{DF}$ & $\mathrm{T}$ & $\mathrm{PT}$ \\
\hline 1 & $\overline{\mathrm{N}} \mathrm{EW} * \mathrm{TRT}$ & & 9 & $\overline{7} .04 \overline{3} 98617$ & $3.1671 \overline{2} 877$ & 4 & 2.22 & $0 . \overline{0} 902$ \\
\hline 2 & $\mathrm{NEW} * \mathrm{TRT}$ & & 8 & 5.02676385 & 2.97686850 & 4 & 1.69 & 0.1666 \\
\hline 3 & $\mathrm{NEW} \star \mathrm{TRT}$ & & 1 & 2.95032645 & 3.16712877 & 4 & 0.93 & 0.4043 \\
\hline 4 & $\mathrm{NEW} * \mathrm{TRT}$ & & 2 & 2.55907978 & 2.97686850 & 4 & 0.86 & 0.4384 \\
\hline 5 & $\mathrm{NEW} * \mathrm{TRT}$ & & 3 & 2.16783311 & 3.16712877 & 4 & 0.68 & 0.5313 \\
\hline 6 & $\mathrm{R} 1$ (REP) & 1 & & 0.67266270 & 1.85885818 & 4 & 0.36 & 0.7358 \\
\hline 7 & REP & 1 & & 0.00000000 & • & & $\cdot$ & . \\
\hline 8 & REP & 2 & & 0.00000000 & $\cdot$ & & - & . \\
\hline 9 & REP & 3 & & 0.00000000 & $\cdot$ & & • & . \\
\hline 10 & $\mathrm{C} 1$ (REP) & 1 & & 0.00000000 & . & & . & . \\
\hline 11 & $\mathrm{C} 1$ (REP) & 2 & & 0.00000000 & . & & $\cdot$ & . \\
\hline 12 & $\mathrm{C} 1$ (REP) & 3 & & 0.00000000 & . & & . & . \\
\hline 13 & $\mathrm{NEW} \star \mathrm{TRT}$ & & 10 & 0.00000000 & 5.74139295 & 4 & 0.00 & 1.0000 \\
\hline 14 & $\mathrm{NEW} * \mathrm{TRT}$ & & 11 & 0.00000000 & 5.74139295 & 4 & 0.00 & 1.0000 \\
\hline 15 & $\mathrm{NEW} \star \mathrm{TRT}$ & & 12 & 0.00000000 & 5.74139295 & 4 & 0.00 & 1.0000 \\
\hline 16 & $\mathrm{NEW} * \mathrm{TRT}$ & & 13 & 0.00000000 & 5.74139295 & 4 & 0.00 & 1.0000 \\
\hline 17 & $\mathrm{NEW} * \mathrm{TRT}$ & & 14 & 0.00000000 & 5.74139295 & 4 & 0.00 & 1.0000 \\
\hline 18 & $N E W * T R T$ & & 15 & 0.00000000 & 5.74139295 & 4 & 0.00 & 1.0000 \\
\hline 19 & $\mathrm{R} 1$ (REP) & 3 & & -0.63974353 & 1.85885818 & 4 & -0.34 & 0.7481 \\
\hline 20 & $\mathrm{NEW}^{\star} \mathrm{TRT}$ & & 6 & -1.88923088 & 3.16712877 & 4 & -0.60 & 0.5830 \\
\hline 21 & $\mathrm{NEW} * \mathrm{TRT}$ & & 5 & -4.84397244 & 2.97686850 & 4 & -1.63 & 0.1790 \\
\hline 22 & $\mathrm{R} 1$ (REP) & 2 & & -5.08002904 & 1.85885818 & 4 & -2.73 & 0.0523 \\
\hline 23 & $\mathrm{NEW} * \mathrm{TRT}$ & & 7 & -5.21607204 & 3.16712877 & 4 & -1.65 & 0.1749 \\
\hline 24 & $\mathrm{NEW} \star \mathrm{TRT}$ & & 4 & -7.79871400 & 3.16712877 & 4 & -2.46 & 0.0695 \\
\hline
\end{tabular}

/* The above are solutions for the random effects arranged in descending order. The intercept plus the above solutions results in the mean for the effect. */

COMMNT

It is possible to construct augmented lattice rectangle designs by the above method. Since the maximum number of new entries $\mathrm{n}$ can be included in an augmented lattice square design, it would appear that an ALSD would be a more useful design.

Another method for constructing these ALSDs is to start with the plan for a balanced lattice square with $r=k+1$ complete blocks. The delete all numbers above $2 \mathrm{k}$ (or $3 \mathrm{k}$ ) leaving only the numbers from 1 to $2 \mathrm{k}$. There are $r=k-1$ complete blocks which have two of the numbers 1 to $2 \mathrm{k}$ in each row and in each column. These form an ALSD.

\section{LITERATURE CITED}

Federer, W. T. (1995). A simple procedure for constructing experiment designs with incomplete blocks of sizes 2 and 3. Biometrical Journal 37(8):899-907. 